\title{
GENERATING FRACTAL LANDSCAPES BY USING FAST FOURIER TRANSFORM
}

\author{
Mushtaq Kareem \\ College of Education, Karbelaa University.
}

\section{Abstract}

This paper aims to provide an introduction to creating fractal landscapes. we will present fast Fourier transform method for generating landscapes. The properties of fractal landscapes and real landscapes that make them fractals are discased.

The purpose of this paper describe algorithms for generating fractal landscapes. This paper also discuss some useful modifications and extensions to the algorithm.

\section{Introduction}

The goal in generating fractal landscapes is to make them look as realistic as possible. A generated landscape can be made quite realistic by adding colour, proper lighting, water, plants, atmospheric effects and other such things. This paper focuses on the process of creating the topological form of the landscape.

The output of the algorithm discussed is a set of altitudes assigned to a two-dimensional grid. Inputs into the algorithm are parameters that define certain desired characteristics of the generated landscape, mainly its roughness.

The algorithm must use randomness otherwise we would not have interesting or unique results. Simply assigning a random altitude to each point in our grid won't do because we expect that two points that are close to each other are likely to have similar altitudes. So we need an algorithm that will take into account the proximity of two points in assigning their altitude. To understand the fractal landscape algorithms, we must first understand why a real landscape is a fractal.

\section{Fractal Landscapes}

Firstly, landscapes are self-similar. They are not self-similar in the sense that looking at a small portion of the landscape, you will see the same shape that you see looking at the entire landscape. Looking at a landscape at different scales, though, you will notice that it exhibits the same basic characteristics at every scale. Consider a mountainous terrain see Fig.(1), each mountain likely has several peaks. Think of these peaks as being the result of a smaller mountainous terrain imposed on top of one mountain in the larger terrain [1] [4]. Further, each of these peaks contains more peaks but on a smaller scale. It is in this sense that landscapes are self-similar.

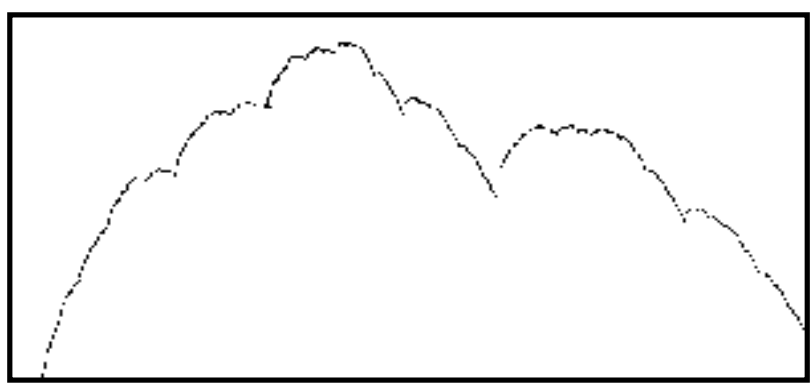

Fig. (1) : The Mountains.

The characteristic that allows us to define landscapes as fractals is the fact that they have fractional dimensions. In 1967, Benoit Mandelbrot coined the popular question: "How long is the coast of Britain?" The answer is not as simple as it may appear. A measurement of the length of the coastline would depend entirely on the scale of the measurement device used [3]. If you were to measure the coastline one kilometer at a time, you may think it would yield a reasonably accurate measurement. But you would have been ignoring all the rough detail within each kilometer. You would find that taking a meter stick and measuring the coastline one meter at a time would yield a far greater result. In fact, every measurement using a smaller scale would yield a greater result, without limit. The answer to the question is that the coastline of Britain does not have a length because it is not one-dimensional. It is a fractal [4].

The algorithm that will be examined make use of the fractal properties of landscapes in converting random numbers into landscapes. The Fourier Transform algorithm uses discrete Fourier transforms to represent a landscape in 
the frequency domain. By moulding the frequencies, this algorithm can convert a set of random numbers into a landscape.

\section{Fast Fourier Transform}

A fast Fourier transform (FFT) is an efficient algorithm to compute the discrete Fourier transform (DFT) and its inverse [6]. FFTs are of great importance to a wide variety of application, from digital signal processing and solving partial differential equation to algorithms for quick multiplication of large integers. This article describes the algorithms, of which there are many; see discrete Fourier transform for properties and applications of the transform [2].

Let $\mathrm{X}_{\mathrm{O}}, \ldots, \mathrm{X}_{\mathrm{N}-1}$ be complex numbers. The DFT is defined by the formula

$$
X_{k}=\sum_{n=0}^{N-1} x_{n} e^{-\frac{2 \pi i}{N} n k} k=0, \ldots, N-1 .
$$

Evaluating these sums directly would take $O\left(N^{2}\right)$ arithmetical operation. An FFT is an algorithm to compute the same result in only $O(N \quad \log N)$ operation. In general, such algorithms depend upon the factorization of $N$, but (contrary to popular misconception) there are FFTs with $O(N \log N)$ complexity for all $N$, even for prime $N$.

Many FFT algorithms only depend on the fact that $\mathrm{e}^{-\frac{2 \pi \mathrm{i}}{\mathrm{N}}}$ is an $N$ th primitive root of unity, and thus can be applied to analogous transform over any finite field, such as number - theoretic transforms.

Since the inverse DFT is the same as the DFT, but with the opposite sign in the exponent and a $1 / \mathrm{N}$ factor, any FFT algorithm can easily be adapted for it as well [5].

\section{Fourier Transform Algorithm}

A Fourier transform is based on the idea that a function can be expressed as a sum of sine or cosine waves at different frequencies. The Fourier transform expresses a function in this form; it converts the function to its frequency domain. A Discrete Fourier Transform (DFT) is a Fourier Transform applied to a set of discrete values. The result is a set of discrete complex numbers of the same size as the original set. The resulting values can be thought of as amplitudes of various frequencies in the original set [6]. The process can be reversed without any change to the original set. A DFT can be performed in $O(N \cdot \log (N))$ using a Fast Fourier Transform (FFT) algorithm making it a reasonably efficient process. A DFT can also be applied to multiple dimensions.

The idea of a Fourier Transform ties into fractal landscapes by thinking of a landscape as a sum of waves at different frequencies. Observe that the amplitude of these waves has a decreasing trend as the frequency increases. This is essentially the same as the observation used in the previous algorithms that the size of perturbations should decrease as the scale at which they are being applied decreases.

The Fourier Transform Algorithm is quite different from the previous algorithms discussed, mainly because it is not an iterative process. At a high level it is quite simple:

1. Create a 2-dimentional grid of random values.

2. Apply a 2-dimentional FFT.

3. Scale each of the values in the transform by $1 / f r$, where $f$ is the frequency represented by that value, and $r$ is the roughness parameter.

4. Apply the inverse FFT. The result is a fractal landscape.

The resulting fractal landscape does not contain any artificial ridges or peaks as the previous algorithms did. In addition, the landscape has the interesting (and sometimes desirable) property that it can be tiled. One side of the landscape flows perfectly into the opposite side.

\section{Conclusion}

We have examined the Fourier transform algorithm for generating fractal landscapes. The Fourier Transform algorithm takes advantage of the observation that frequencies can be used to describe a natural landscape. It has the property that a generated landscape can be tiled. It can also be modified to create mountain range-like landscapes.

The landscapes generated by the Fourier transform algorithms described fail to mimic certain characteristics of real landscapes. An easy way to create a more realistic fractal landscape is to multiply two generated landscapes together, creating smoother valleys 
and rougher peaks. More advanced methods exist to convert a generated landscape into a realistic landscape such as simulating the erosion process.

\section{Future Work}

1. Collecting between the Fast Fourier transform algorithms and another algorithms, such as, the Cooley-Tukey algorithm, Multidimensional FFT algorithms to generating best value of fractal landscapes.

2. Using this subject in coding theory, so as to use a fractal landscape as a password to the systems.

\section{References}

[1] Barnsley, M. F, "Fractals Everyweary", Academic Press Inc., New York, (1993).

[2] "Fast Fourier Transform." Wikipedia. 22 November 2006. http://en.wikipedia.org/wiki/.

[3] Mahmood B.; "Processor Design for Fractal Signal", Ph.D. Thesis, University of Technology, (1996).

[4] Mushtaq K.; "Solution of Inverse problem of fractal image using optimization method", MSc. Thesis, Al-Nahrien University, August (2002).

[5] N. Brenner and C. Rader, A New Principle for fast Fourier Transformition; IEEE Acoustics, Speech \& Signal Processing 24: 264-266.

[6] W. E. Williams; Fourier Series and Boundary - Value Problems; Page Bros (Norwich) Ltd., Norwich, 1973.

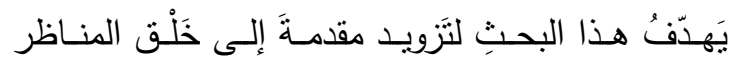

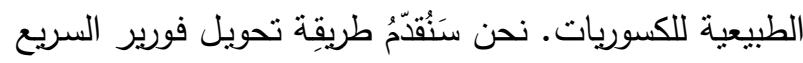

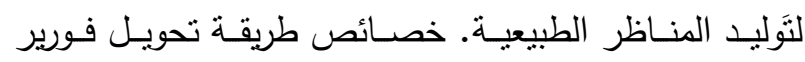
السـريع وخصـائص المنـاظر الطبيعيـة الحقيقيـة التي تَجْعُلُهم كسوريات نوقشت.

الغرضُ من هذا البحث هو وصف الخوارزمية لتَوليد المناظر

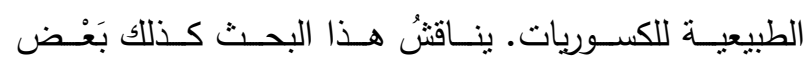
التعديلاتِ المفيدةِ وإمتداداتِ إلى الخوارزميةِة. 